\title{
REPRODUCTIVE ENDOCRINOLOGY DMPA SUPPRESSES RESPONSE TO HIV-1
}

Use of depot medroxyprogesterone acetate (DMPA) weakens both innate and adaptive immune responses to HIV type 1 (HIV-1) and, therefore, reduces host resistance to the infection, new data suggest.

DMPA is a progestin-only, injectable hormonal contraceptive that is usually administered at 3-monthly intervals. The use of DMPA is popular in subSaharan Africa and in other areas with a high prevalence of HIV-1 infection. Although this type of contraception is effective and affordable, a number of observational studies have found an association between the use of hormonal contraception, in particular DMPA, and an increased risk of the acquisition and transmission of HIV-1. Huijbregts and co-workers set out to investigate the immunological mechanisms underlying the effect of DMPA on HIV-1 acquisition.

Using peripheral blood mononuclear cells from healthy women, the researchers show that, in vitro, DMPA inhibits the production of cytokines by activated $T$ cells, including IFN- $\gamma$, IL-2, IL-4, IL-6, IL-12, TNF and CCL3. DMPA also reduced the capacity of plasmacytoid dendritic cells -which are involved in early recognition of viral and bacterial infections- to respond to activation by ligands of Toll-like receptors 7,8 and 9. Specifically, DMPA reduced the production of IFN- $\alpha$ and TNF by these activated cells.

An in vivo experiment also indicated an effect of DMPA use on the immune system. In 19 women using DMPA, significantly lower levels of IFN-a were found in plasma and genital secretions than in 21 women not using any form of hormonal contraception. The findings of additional in vitro experiments demonstrated that DMPA prevents the downregulation of the HIV-1 co-receptors CXCR4 and CCR5 on activated $T$ cells and increases the rate of HIV-1 proliferation.

The investigators say that their findings argue against the continuous use of high-dose DMPA in women at high risk of HIV-1 infection, although they stress the importance of safe and effective contraception in these women. They propose gradual replacement of DMPA with other modern types of contraception that have less of an affect on the immune system.

Carol Wilson

Original article Huijbregts, R. P. et al. Hormonal contraception and HIV-1 infection: medroxyprogesterone acetate suppresses innate and adaptive immune mechanisms. Endocrinology doi:10.1210/en.2012-1850 